Applied Mathematical Sciences, Vol. 9, 2015, no. 94, 4653 - 4659

HIKARI Ltd, www.m-hikari.com

http://dx.doi.org/10.12988/ams.2015.56429

\title{
Dynamic Model of Space Robot Manipulator
}

\author{
Polina Efimova
}

Saint-Petersburg State University, Applied Mathematics and Control Processes

Universitetskii prospect 35, Petergof, Saint-Petersburg, Russia, 198504

\section{Dzmitry Shymanchuk}

Saint-Petersburg State University, Applied Mathematics and Control Processes Universitetskii prospect 35, Petergof, Saint-Petersburg, Russia, 198504

Copyright (C) 2015 Polina Efimova and Dzmitry Shymanchuk. This article is distributed under the Creative Commons Attribution License, which permits unrestricted use, distribution, and reproduction in any medium, provided the original work is properly cited.

\begin{abstract}
In this study the dynamic model of a space robot manipulator is constructed. The space robot manipulator as a three-degree-of-freedom manipulator on a movable base is represented. Functional performance of space robotic manipulator near the orbital space station is considered. It is also anticipated that the motion occurs in a weightless environment without affecting the dissipative forces. Based on this model of space robot manipulator the first problem of manipulator dynamics is solved. The problem is solved using the expansion pack Symbolic Math Toolbox of mathematical package Matlab.
\end{abstract}

Keywords: space robot manipulator, dynamic model, modeling, simulation, first problem of manipulator dynamics, control problem

\section{Introduction}

Consider space robot manipulator as a controlled system consisting of a freeflying base and a manipulator or a system of manipulators mounted on it (Fig. 1). Recently, it has called more attention of researchers not only for its performance capabilities, but also rapid development of applications of this kind of technical systems in space. However, the research methods for 

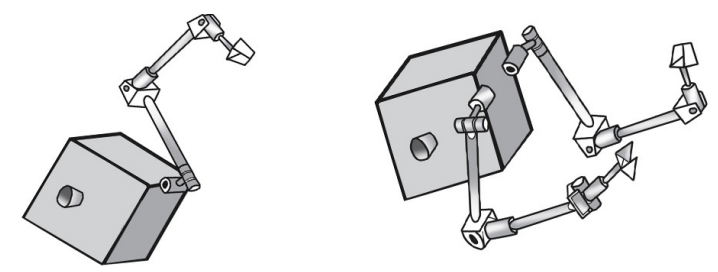

Figure 1: Schematic representation of space robot manipulators

ground robotic manipulators are not fully appropriate for applying to space ones mainly because of its dynamical. In the study of considered class of objects it is necessary to take into account that the robot's motion takes place in conditions of weightlessness that allows to neglect the force of gravity. Besides, the robot operates on a high enough orbit, which allows to neglect the environmental resistance, as well. The absolutely free-flying base of the robot, which entails an additional degree of mobility significantly complicates the things. In this regard, the study of such class of robot manipulators should be considered in the context of a new, separate scientific and technical concept $[1-4]$.

\section{$2 \quad$ Kinematic scheme}

In this paper, the model of three-link space robot manipulator is considered. Its motion takes place in the vicinity of an orbital space station. The kinematic scheme for the described mechanical system is shown on Fig. 2. The movement

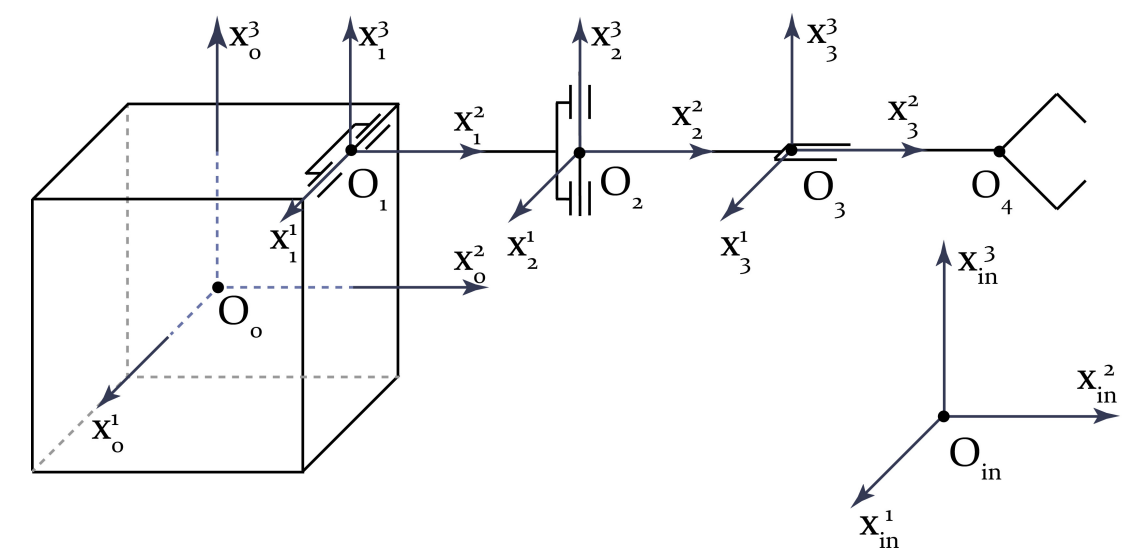

Figure 2: Kinematic scheme of space robot manipulator

is considered in inertial coordinate system $O_{i n} x_{i n}^{1} x_{i n}^{2} x_{i n}^{3}$ associated with the orbital space station. On Fig. 3 the generalized coordinates of the robot are shown. They clearly define the position of the mechanical system in absolute space. 


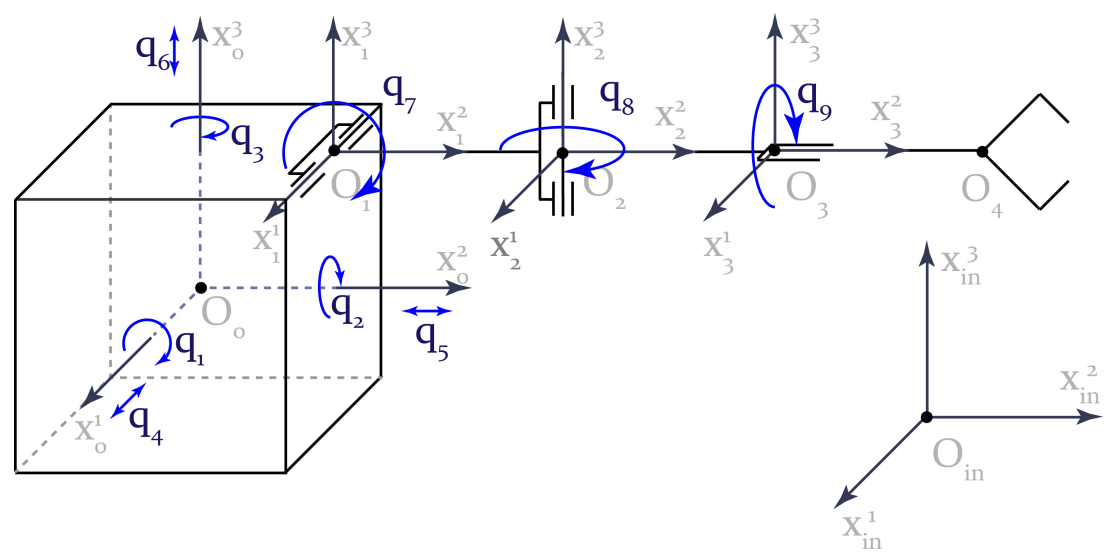

Figure 3: Generalized coordinates of space manipulation robot

\section{Determination of generalized velocities}

To build a dynamic model of space robot it is necessary to define a connection between its generalized velocities and linear and angular ones. For this purpose the theorem of angular velocities addition and the theorem of velocities in complex movement addition are used. Then the equations of constraints between the generalized velocity vector of the robot and its linear and angular velocities are determined by formulas [3]:

$$
\left[\begin{array}{c}
\omega_{i}^{1} \\
\omega_{i}^{2} \\
\omega_{i}^{3}
\end{array}\right]_{i}=\Omega_{i}\left[\begin{array}{c}
\dot{q}_{1} \\
\vdots \\
\dot{q}_{9}
\end{array}\right], \quad\left[\begin{array}{c}
v_{i}^{1} \\
v_{i}^{2} \\
v_{i}^{3}
\end{array}\right]_{i}=V_{i}\left[\begin{array}{c}
\dot{q}_{1} \\
\vdots \\
\dot{q}_{9}
\end{array}\right]
$$

where index $i$ outside the brackets indicates the $i$-th body of the robot when considering the angular velocity or a point $O_{i}$ in case of linear velocity; $\omega_{i}^{1}, \omega_{i}^{2}, \omega_{i}^{3}$ are the angular velocity projections of the $i$-th body in the coordinate system $O_{i} x_{i}^{1} x_{i}^{2} x_{i}^{3} ; v_{i}^{1}, v_{i}^{2}, v_{i}^{3}$ are the linear velocity projections of the point $O_{i}$ on the axis of the same system of coordinates; $\Omega_{i}$ and $V_{i}$ are the constraint matrices of angular and linear robot's velocity with the vector of the generalized velocities determined by the following recurrence relations (under the assumption that $\cos (q)=C q, \sin (q)=S q)[3]$ :

$$
\begin{gathered}
\Omega_{i}=\alpha_{i, i-1} \Omega_{i-1}+I_{i}^{\omega}, \quad V_{i}=\alpha_{i, i-1} V_{i-1}-\alpha_{i, i-1} L_{i-1} \Omega_{i-1}+I_{i}^{v}, \\
\Omega_{0}=\left[\Omega_{* 0} \vdots O_{3 \times 6}\right], \Omega_{* 0}=\left[\begin{array}{ccc}
0 & S q_{1} S q_{3} & C q_{1} \\
0 & C q_{1} S q_{3} & -S q_{1} \\
1 & C q_{3} & 0
\end{array}\right], \quad V_{0}=\left[O_{3 \times 3} \vdots \alpha_{0} \vdots O_{3 \times 3}\right],
\end{gathered}
$$

where the matrices $\alpha_{i, i-1}$ are of the following form: 


$$
\begin{gathered}
\alpha_{1,0}=\left[\begin{array}{ccc}
1 & 0 & 0 \\
0 & C q_{7} & -S q_{7} \\
0 & S q_{7} & C q_{7}
\end{array}\right], \alpha_{2,1}=\left[\begin{array}{ccc}
C q_{8} & -S q_{8} & 0 \\
S q_{8} & C q_{8} & 0 \\
0 & 0 & 1
\end{array}\right], \alpha_{3,2}=\left[\begin{array}{ccc}
C q_{9} & 0 & S q_{9} \\
0 & 1 & 0 \\
-S q_{9} & 0 & C q_{9}
\end{array}\right], \\
\alpha_{0}=\left[\begin{array}{ccc}
C q_{2} C q_{3}-S q_{2} C q_{1} S q_{3} & S q_{3} C q_{2}+C q_{3} C q_{1} S q_{2} & S q_{1} S q_{2} \\
-C q_{3} S q_{2}-S q_{3} C q_{1} C q_{2} & -S q_{2} S q_{3}+C q_{2} C q_{1} C q_{3} & S q_{1} C q_{2} \\
S q_{3} S q_{1} & -C q_{3} S q_{1} & C q_{1}
\end{array}\right] .
\end{gathered}
$$

Matrices $L_{i}, i=0, \ldots, 3$ characterizing the geometric parameters of the robot are determined as follows:

$$
L_{i}=\left[\begin{array}{ccc}
0 & 0 & l_{i} \\
0 & 0 & 0 \\
-l_{i} & 0 & 0
\end{array}\right], \quad L_{0}=\left[\begin{array}{ccc}
0 & -x_{0}^{3} & x_{0}^{2} \\
x_{0}^{3} & 0 & 0 \\
-x_{0}^{2} & 0 & 0
\end{array}\right]
$$

where $l_{i}$ is a length of the $i$-th segment, $x_{0}^{2}, x_{0}^{3}$ are coordinates of the point $O_{1}$ in coordinate system constrained with the body of robot.

To simplify the form of the equations the concept of quasivelocity can be used. Quasivelocity of the $i$-th body is a $(6 \times 1)$-dimensional vector. Its first three components are the angular velocity projections of the $i$-th body on the axis of the coordinate system constrained with this body, the next three components define the linear velocity projection of point $O_{i}$ on the axis of the same coordinate system:

$$
\dot{\mathbf{x}}_{i}=\left[\begin{array}{llllll}
\omega_{i}^{1} & \omega_{i}^{2} & \omega_{i}^{3} & v_{i}^{1} & v_{i}^{2} & v_{i}^{3}
\end{array}\right]_{i}^{\mathrm{T}} .
$$

In this case the quasivelocity the entire system is a $(24 \times 1)$ - dimension vector which contains quasivelocities of all the system bodies taken in the order of numbering the bodies:

$$
\dot{\mathbf{x}}=\left[\begin{array}{lllllll}
\omega_{0}^{1} & \ldots & v_{0}^{3} & \ldots & \omega_{3}^{1} & \ldots & v_{3}^{3}
\end{array}\right]^{\mathrm{T}} .
$$

Then the equations of constraint between the angular and linear velocities of the system and the generalized velocities vector can be written in the following form:

$$
\dot{\mathbf{x}}=C \dot{\mathbf{q}}, C=\left(\Omega_{0}, V_{0}, \Omega_{1}, V_{1}, \Omega_{2}, V_{2}, \Omega_{3}, V_{3}\right)^{\mathrm{T}}
$$

\section{Dynamic model}

To derive the equations of the robot motion in the form of Lagrange equations of the second kind

$$
\frac{d}{d t} \frac{\partial T}{\partial \dot{\mathbf{q}}}-\frac{\partial T}{\partial \mathbf{q}}=\mathbf{Q}
$$


where the vector $\mathbf{Q}$ contains a vector of control forces and moments it is necessary to determine the kinetic energy of the system obtained by summing the kinetic energies of all the system objects:

$$
T=\sum_{i=0}^{3} T_{i}
$$

where $T_{i}$ is the kinetic energy of the $i$-th body. In this case, the total kinetic energy of the system is determined by the formula

$$
T=\frac{1}{2} \dot{\mathbf{x}}{ }^{\mathrm{T}} B \dot{\mathbf{x}}
$$

where $\dot{\mathbf{x}}$ is a system quasivelocity vector and the matrix $B$ describes the massinertial characteristics of the robot.

The substitution of the known quasivelocity vector to the expression of the kinetic energy with the use of the generalized velocities vector gives a formula defining the kinetic energy of the system in terms of generalized coordinates and generalized velocities [5]:

$$
T=\frac{1}{2} \dot{\mathbf{q}}^{\mathrm{T}} A(\mathbf{q}) \dot{\mathbf{q}}, A=C^{\mathrm{T}} B C .
$$

Then the substitution of the obtained expression for the kinetic energy to the Lagrange equation of the second kind and consistent differentiating of the term gives the following result

$$
\begin{gathered}
A(\mathbf{q}) \ddot{\mathbf{q}}+\sum_{s=1}^{9}\left(\dot{\mathbf{q}}^{\mathrm{T}} D_{s}(\mathbf{q}) \dot{\mathbf{q}}\right) \mathbf{e}_{s}=\mathbf{Q} \\
D_{s}(\mathbf{q})=\left(d_{i t}^{s}(\mathbf{q})\right), \quad i=1, \ldots, n, \quad t=1, \ldots, n, \quad s=1, \ldots, n, \\
d_{i t}^{s}(\mathbf{q})=\frac{1}{2}\left(\frac{\partial a_{i s}}{\partial q_{t}}+\frac{\partial a_{t s}}{\partial q_{i}}-\frac{\partial a_{i t}}{\partial q_{s}}\right)
\end{gathered}
$$

where $\mathbf{e}_{s}$ is a unit $(9 \times 1)$-dimesional column vector with $s$-th nonzero component.

\section{Example}

To implement the desired motion of the considered mechanical system it is necessary to determine the values of generalized forces applied to the base and in the joints. For this purpose the solution of the first problem of the manipulator dynamics is represented. 
Let the generalized coordinates change due to the following law

$$
q_{i}(t)=q_{i}\left(t_{0}\right)+\lambda(t)\left(q_{i}\left(t_{1}\right)-q_{i}\left(t_{0}\right)\right),
$$

where $t_{0}$ is initial time, $t_{1}$ is finite time, $\lambda(t)$ satisfies the condition $\lambda\left(t_{0}\right)=$ $0, \lambda\left(t_{1}\right)=1$. In this case, the value $\dot{\lambda}(t)$ defines the velocity of change of the generalized coordinates.

Consider $\dot{\lambda}=\frac{2}{\Delta t} \sin ^{2}\left(\frac{\pi t}{\Delta t}\right)$, where $\Delta t=t_{1}-t_{0}$. Such value of the velocity provides a smooth movement of manipulator along the entire trajectory. In the simulation the following initial and finite values for the generalized coordinates were used:

$$
\mathbf{q}\left(t_{0}\right)=(0,0,0,0,0,0,0,0,0)^{\mathrm{T}}, \quad \mathbf{q}\left(t_{1}\right)=\left(0,0, \frac{\pi}{2}, 6,-6,4, \frac{\pi}{2}, \frac{\pi}{2},-\frac{\pi}{2}\right)^{\mathrm{T}} .
$$

Graphics of the generalized forces changes are plotted using the mathematical package Matlab (Fig. 4) when $t_{0}=0$ and $t_{1}=60$.

a)

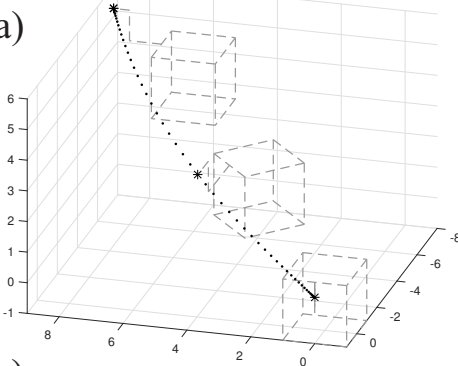

c)

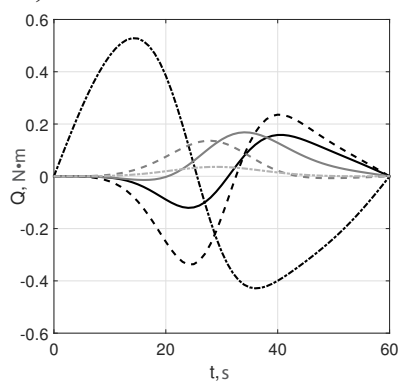

b)

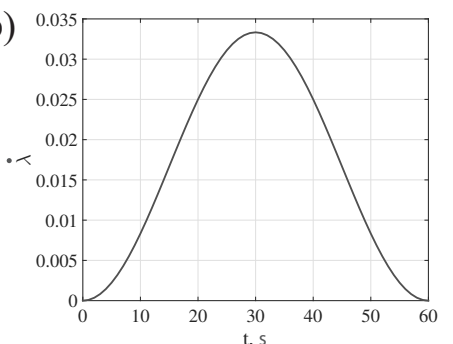

d)

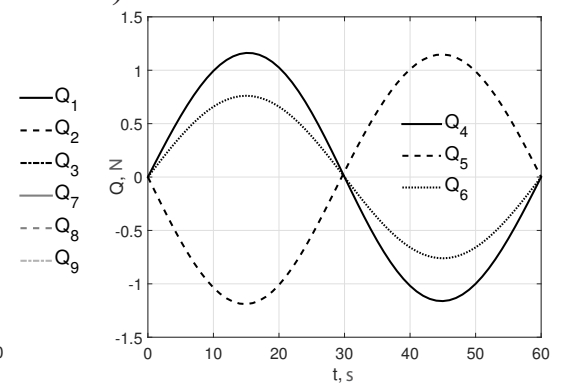

Figure 4: a) trajectory of characteristic point; b) velocity profile; c) graphics of generalized forces changes $\left.Q_{i}, i=1,2,3,7,8,9 ; \mathrm{d}\right)$ graphics of generalized forces changes $Q_{i}, i=4,5,6$

\section{Conclusion}

The dynamic model for the given kinematic scheme of the three-link space robot manipulator is obtained. The solution of the first problem of the manipulator dynamics is represented by the example of a smooth movement of the 
robot along the entire trajectory. The simulation of the motion is implemented using the mathematical package Matlab.

\section{Acknowledgment}

The authors acknowledge Saint-Petersburg State University for a research grant 9.37.345.2015.

\section{References}

[1] F. M. Kulakov, A. S. Shmyrov, D. V. Shymanchuk, Supervisory remote control of space robot in an unstable libration point, Proceedings of the 2013 IEEE rth International Conference on Intelligent Data Acquisition and Advanced Computing Systems, IDAACS, 2 (2013), 925-928. http://dx.doi.org/10.1109/idaacs.2013.6663062

[2] A. Shmyrov, V. Shmyrov, D. Shymanchuk, Prospects for the use of space robots in the neighborhood of the libration points, Applied Mathematical Sciences, 8 (2014), 2465-2471. http://dx.doi.org/10.12988/ams.2014.43158

[3] F. M. Kulakov, Supervisory control by robotic manipulator, Moscow, Nauka, 1980, 448 p. (in Russian)

[4] V.Yu. Rutkovsky, V.M. Suhanov, V. M. Glumov, Equations of motion and control of Free Space manipulation robot in reconfiguration mode, Automation and telemechanics, 1 (2010), 80-98. (in Russian)

[5] K. S. Fu, R. C. Gonzalez, C. S. G. Lee, Robotics: Control, Sensing, Vision, and Intelligence, McGraw-Hill International Editions, 1987, 580 p.

Received: June 15, 2015; Published: July 2, 2015 